\title{
THE IMPACT OF PERSONAL PRODUCTIVITY DUE TO THE ATTACKS OF CORONAVIRUS DISEASE 2019: USING PARTIAL LEAST STRUCTURAL EQUATION MODEL
}

\author{
SHEKH ABDULLAH-AL-MUSA AHMED ${ }^{1 *}$, NIK ZULKARNAEN BIN KHIDZIR ${ }^{1}$, SHIRIN AFROZ
}

${ }^{1}$ Department of, Faculty of Creative Technology and Heritage, Universiti Malaysia Kelantan, Malaysia. ${ }^{2}$ Chittagong Diabetic General Hospital, Chittagong, Bangladesh. Email: almusa.c17e002f@siswa.umk.edu.my

Received: 27 august 2020, Revised and Accepted: 29 september 2020

\begin{abstract}
Global coronavirus disease 2019 (COVID-19) is an infectious disease caused by severe acute respiratory syndrome coronavirus 2 (SARS-CoV-2). The disease was first identified in December 2019 in Wuhan, the capital of China's Hubei Province, and has since spread globally, resulting in the ongoing 2019-2020 coronavirus pandemic that has an impact which would be disturbed the personal productivity in the community. The dependent variable which is threat of COVID-19 (Thr_Cov-19), vulnerability of COVID-19 (Vul_Cov-19), and the unexpected change in society factors and one independent variable that is personal disturbance factors is used in this paper. Moreover, using as an indicator which will determine disturbance of personal productivity (D_PP) in the society. Since multiple regression by partial least square-structural equation modeling is used to examined of data by following unstructured method. Moreover, the resulting point out three dependent variables significantly influences on the independent variable of personal productivity in the society. As a matter of fact, this study concludes the foremost influence factor on D_PP in society due to COVID-19 risk factors such as Thr_Cov-19, Vul_Cov-19, and unexpected changed in the society factors. This study contributes to introductory study but vibrant understanding in stimulating the prediction of personal productivity in the society due to the COVID-19 attacks.
\end{abstract}

Keywords: Coronavirus disease, Threat of COVID-19, Vulnerability of COVID-19, Unexpected changed in the society, Personal productivity.

\section{INTRODUCTION}

The common symptoms of coronavirus disease 2019 (COVID-19) include fever, cough, and shortness of breath. Other symptoms may include fatigue, muscle pain diarrhea, sore throat, loss of smell, and abdominal pain. While the majority of cases result in mild symptoms, some progress to viral pneumonia and multiorgan failure. As of April 6,2020 , more than $1,270,000$ cases of have been reported in more than 200 countries and territories, resulting in more than 69,500 deaths. More than 264,000 people have recovered. The increasing amount of COVID-19 cases in the society also resulting the effect of the personal productivity [1].

As of April 11, 2020, more than 1.71 million cases have been reported in more than 200 countries and territories, resulting in more than 103,000 deaths. More than 388,000 people have recovered. The virus is mainly spread between people during close contact, often through small droplets produced during coughing, sneezing, or talking [2].

While these droplets are produced when breathing out, they usually fall to the ground or surfaces rather than being infectious over large distances. People may also become infected by touching a contaminated surface and then their face. The virus can survive on surfaces for up to $72 \mathrm{~h}$ [3].

Coronavirus is most contagious during the first 3 days after onset of symptoms, although spread may be possible before symptoms appear and in later stages of the disease. The COVID-19 outbreak is still evolving globally and remains a Public Health Emergency of International Concern [4]. Like many other member states around the world, Bangladesh is preparing to respond to additional cases and possible widespread transmission of the disease [3]. As cases of COVID-19 continue to climb, public concern in Bangladesh regarding the severity of the disease and population vulnerability of COVID-19 (Vul_Cov-19) is also growing. Hence, the threat of COVID-19 (Thr_Cov-19) variable occurred when most people who develop COVID-19 and seek medical attention will not be tested for it, at least not early on, because of the shortage of testing kits and confusion around where to send people who are showing symptoms [3].
Whereas the theory of COVID-19 attacks in showing the disturbance of personal productivity (D_PP) variable such as creative activity. However, the internet connection all over the world may cause the vulnerability of the people, sometimes people get wrong information. Since the coronavirus has incited a lot of change across the world, these last couple months. It is also indicated the variable of unexpected change in society due to COVID-19 attacks (Unxch_Cov-19). Entire cities have shut down, trains and airfare have all but nearly stopped, public spaces are shut down, concerts cancelled, and ski resorts closed [5].

\section{LITERATURE REVIEW}

In this section, analyzing of theory of personal productivity due to COVID-19 virus attacks in the society and literature related to hypothesis which would be development and discussed.

\section{Theory of D_PP}

In this article, it is shown that three constructs which are related with personal productivity due to COVID-19 attacks having the D_PP in the society. The theory of D_PP is the independent variable. While scrolling on social media amid the COVID-19 pandemic, may have noticed the push for less productivity. Many researchers found out that mostpeople tend to curate themselves and could not put their best self on social activities. Seeing the mistakes and the flubs and the errors. Seeing what people want the world to see about them [6].

Hence, it is really important right now for people to realize that this is new for everyone. There is no road map on how to manage a pandemic. Thus, the following risk factors caused D_PP factors that play a significance role in measure toward the personal disturbance of productive in the society [5].

\section{The Thr_Cov-19}

The Thr_Cov-19 factors is indicating that not all who are infected develop symptoms, but their role in transmission is unknown. Preliminary evidence suggests asymptomatic cases may contribute to the spread of the disease. The proportion of infected people who do not display symptoms is currently unknown and being studied, with 
the Korea Centers for Disease Control and Prevention (CDC) reporting that $20 \%$ of all confirmed cases remained asymptomatic during their hospital stay. China's National Health Commission began including asymptomatic cases in its daily cases on April 1, of the 166 infections on that day, 130 (78\%) were asymptomatic [7]. In the perspective of D_PP due to the attack of COVID-19, the Thr_Cov-19 factors is salience due to those infected with the virus may be asymptomatic or develop flulike symptoms, including fever, cough, fatigue, and shortness of breath. Emergency symptoms include difficulty breathing, persistent chest pain or pressure, confusion, difficulty waking, and bluish face or lips, immediate medical attention is advised if these symptoms are present Thus, the following hypothesis is developing H1: Thr_Cov-19 factors that play a significance role in disturbing toward the Unxch_Cov-19.

\section{The Vul_Cov-19}

The Vul_Cov-19 is about how the disease spreading is still being determined. The WHO and the US CDC say that it is primarily spread during close contact and by small droplets produced when people cough, sneeze, or talk, with close contact being within 1-3 m (3 ft 3 in- 9 $\mathrm{ft} 10 \mathrm{in}$ ). The virus was present in most patient's saliva in quantities reaching 100 million virus strands per $1 \mathrm{~mL}$. Even it is found that an uncovered cough can lead to droplets travelling up to 4.5 meters $(15$ feet) [8]. During the 2020 pandemic, found that advice on the distance droplets could travel might be based on old 1930s research which ignored the protective effect and speed of the warm moist outbreath surrounding the droplets [9]. Researcher found that an uncovered cough or sneeze can travel up to 8.2 meters ( 27 feet). Thus, the following hypothesis is developing $\mathrm{H}_{2}$ : Vul_Cov-19 factors that play a significance role in disturbing toward the Unxch_Cov-19.

\section{The Unxch_Cov-19}

Actually preventive measures to reduce the chances of infection include staying at home, avoiding crowded places, washing hands with soap and water often and for at least $20 \mathrm{~s}$, practicing good respiratory hygiene, and avoiding touching the eyes, nose, or mouth with unwashed hands and nose with a tissue when coughing or sneezing and recommend using the inside of the elbow if no tissue is available.

Also recommend proper hand hygiene after any cough or sneeze. Social distance strategies aim to reduce contact of infected persons with large groups by closing schools and workplaces, restricting travel, and cancelling large public gatherings [9]. Distancing guidelines also include that people stay at least 6 feet $(1.8$ $\mathrm{m})$ apart. Thus, the following hypothesis is developing H3: The Unxch_Cov-19 factors that play a significance role in disturbing toward the personal productive in the society.

\section{METHODOLOGY}

In this section, the methodology of the research is discussed. In addition, the research conceptual framework in introduced and unstructured secondary data collected and data analyzed to find out the impact of personal disturbance of productivity due to COVID-19 attacks in the society.

\section{Conceptual framework}

A conceptual framework of personal disturbance of productivity due to COVID-19 attacks in the society that synthesis from various literatures on how to explain a phenomenon. It maps out the actions required in the course of the study given the knowledge of relationship of hypothesis that points of view and observations on the subject of the study. Conceptual framework of personal disturbance of productivity due to COVID-19 attacks is a term that is widely used but poorly understood. In general, there is a delay between the moment when a person is infected with the virus and the time when they develop symptoms. This is called the incubation period [9]. The incubation period for COVID-19 is typically 5-6 days but may range from 2 to 14 days. Hence, $97.5 \%$ of people who develop symptoms will do so within 11.5 days of infection. People are managed with supportive care, which may include fluid therapy, oxygen support, and supporting other affected vital organs. The CDC recommends that those who suspect they carry the virus wear a simple face mask. Extracorporeal membrane oxygenation has been used to address the issue of respiratory failure, but its benefits are still under consideration. The reason, the conceptual framework of system consists of concepts, assumptions, expectations, beliefs, and theories that support and inform the researcher [10] (Fig. 1).

\section{Sample characteristics}

In the sample collection section, using unstructured methods and among total 87 questionnaire were 39 returned, so $44 \%$ response rate. A Shapiro-Wilk test $(p>0.05)$ and a visual inspection of their histogram, normal Q. However, Q plots and box plots showed that for the research article. In that case, the Thr_Cov-19, the Unxch_Cov-19, and theory of D_PP were approximately normally distributed, with a skewness of 0.373 (standard error $=0.361$ ) and a kurtosis of 0.583 (standard error $=0.716$ ) [11].

\section{Results and data analysis}

To test the hypothesis, a non-parametric structural equation modeling by partial least square (PLS) analysis is done for the personal disturbance of productivity due to COVID-19 attacks in the society. It is seen that PLS is a suitable approach to find out the impact value. Some medical professionals recommend paracetamol (acetaminophen)

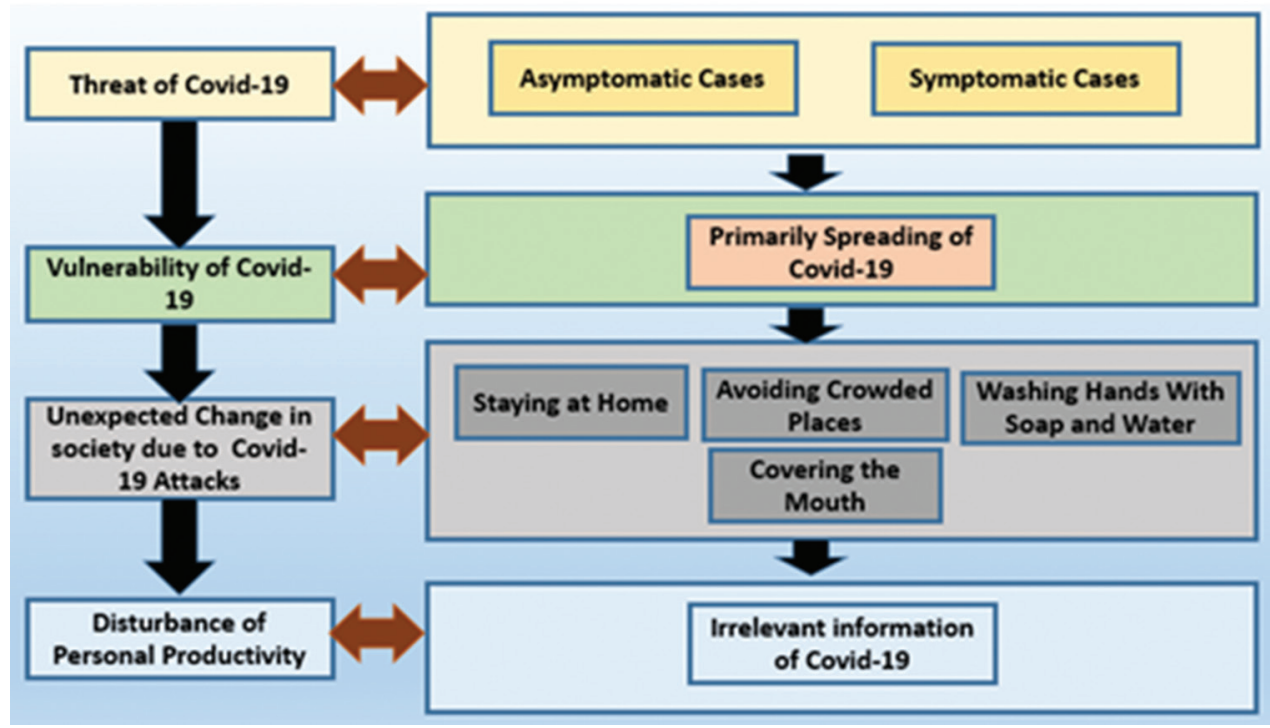

Fig. 1: Conceptual framework of personal disturbance of productivity due to COVID-19 attacks in the society 
over ibuprofen for first-line use. The WHO does not oppose the use of nonsteroidal anti-inflammatory drugs (NSAIDs) such as ibuprofen for symptoms, and currently, there is no evidence that NSAIDs worsen COVID-19 symptoms. And impact showing when this factor variable may effect in the society and how much disturbance might feel when due to COVID-19 attacks [11].

\section{Measurement analysis}

Kaiser-Meyer-Olkin (KMO) and Bartlett's test of sphericity are user to access the factor sample adequacy for analysis. The value of KMO must be greater or equal to 0.6. In this study, the KNO result is considered a good as it is achieved 0.863. While Bartlett's test of sphericity is showing the high significant value $(p<0.001)$ (Wiebke, 2009). Later, variable factors analysis is employed to test the discriminant validity of the items. In the Thr_Cov-19 variable, there are six factors (Thr_Cov-19_1, Thr_Cov-19_2, Thr_Cov-19_3, Thr_Cov-19_4, Thr_Cov-19_5, and Thr_Cov-19_6). For the Vul_Cov-19 possess five factors (Vul_Cov-19_1, Vul_Cov-19_2, Vul_Cov-19_3, Vul_Cov-19_4, and Vul_Cov-19_5).

The Unxch_Cov-19 is four factors (Unxch_Cov-19_1,

Unxch_Cov-19_2, Unxch_Cov-19_3, and Unxch_Cov-19_4). Moreover,

the variable D_PP possessesfourfactors(D_PP_1,D_PP_2,D_PP_3, and

D_PP_4).Questionnaire was designed according the factors name.

When unstructured methods were followed and tried to find out the impact.

But from the factor analysis (Thr_Cov-19_2, Thr_Cov-19_4, Vul_Cov19_3, Vul_Cov-19_5, Unxch_Cov-19_1) has been removed due to the low factor loading $(<0.6)$. Furthermore, the assessment of convergent validity has assessed the value of average variance extracted (AVE) of the variable such as Thr_Cov-19, Vul_Cov-19, and the Unxch_Cov-19 factors. The value of AVE must be above than 0.5. The result in Table 1 demonstrates that convergent validity is satisfied with the threshold value. In addition, to access the discriminant validity, the square root of AVE must be greater than interconstruct correlation (Lowry et al., 2014). The result of discriminant validity is shown in Table 2 which is diagonal value represents the square root of AVE. As a final composite reliability (CR) and Cronbach's alpha coefficient are used to assess reliability, as shown in Table 1 , the CR value of 0.8 , and the Cronbach's alpha is $>0.7$. This result shows that the reliability of construct is reliable (Fig. 2).

\section{PATH MODEL ASSESSMENT}

It is valid to access the multicollinearity issue in the model. As shown in Table 3, VIF value between construct is less than the threshold value (5.0). The value shown in Table 3 demonstrates that the multicollinearity issue is not happening in the model. The result of path assessment is shown in Table 3. The bootstrap technique was done to test the significance of the model.

A sub-sample of 500 is used with 0.05 significance level. The value for path coefficient result as shown in Fig. 3 and Table 3 indicates that the

Table 1: The Cronbach's alpha, CR, and AVE values

\begin{tabular}{llll}
\hline $\begin{array}{l}\text { Coronavirus disease 2019 } \\
\text { (COVID-19) attacking risk variables }\end{array}$ & $\begin{array}{l}\text { Cronbach's } \\
\text { alpha }\end{array}$ & CR & AVE \\
\hline Threat of COVID-19 (Thr_Cov-19) & 0.885 & 0.928 & 0.812 \\
$\begin{array}{l}\text { vulnerability of COVID-19 (Vul_Cov-19) } \\
\text { Unexpected change in society due to }\end{array}$ & 8.723 & 0.843 & 0.663 \\
$\begin{array}{l}\text { COVID-19 attacks (Unxch_Cov-19) } \\
\begin{array}{l}\text { Disturbance of personal productivity } \\
\text { (D_PP) }\end{array}\end{array}$ & 0.665 & 0.922 & 0.707 \\
\hline
\end{tabular}

Table 2: KMO and Bartlett's test of sphericity

\begin{tabular}{|c|c|c|}
\hline \multirow{2}{*}{\multicolumn{2}{|c|}{$\begin{array}{l}\text { KMO measure adequacy sampling } \\
\text { Bartlett's test of approximately. Chi-square }\end{array}$}} & 0.0862 \\
\hline & & 937.88 \\
\hline Sphericity & $\mathrm{DE}$ & 171 \\
\hline & Sig & 0.000 \\
\hline
\end{tabular}

relationship the Unxch_Cov-19. The study expresses a positive and direct impact on countermeasure to D_PP toward the Vul_Cov-19 $(\mathrm{p}<0.001)$. This result indicates that threat value of the system plays a significance role in determining the D_PP in the society [12]. In addition, in the path model, all variable factors are connected to D_PP. Since coronaviruses are a group of related viruses that cause diseases in mammals and birds. In humans, coronaviruses cause respiratory tract infections that can range from mild to lethal. Mild illnesses include some cases of the common cold (which has other possible causes, predominantly rhinoviruses), while more lethal varieties can cause SARS, MERS, and COVID-19. Finally, there is a direct influence between the variable factors toward the D_PP. Since the impact is 0.584 , which is showing the moderate effect happening in the case any due to COVID-19 attacks in the society. As a final point, the coefficient deamination $\mathrm{R}$ squared value for a dependent variable which is the D_PP is $58 \%$ where $\mathrm{p}<0.001$. This endogenous construct manifests a high level of capturing variance which means that it is well predicted by exogenous constructs.

\section{DISCUSSION AND CONCLUSION}

The world faces a severe and acute public health emergency due to the ongoing COVID-19 global pandemic. Hence, the theory of personal productivity has been shown and to investigate the D_PP due to coronavirus disease 2019 (COVID-19) attacking risks such as Thr_Cov19, Vul_Cov-19, and Unxch_Cov-19 factor. For analysis and verification, multiple linear regression analysis was used. Thr_Cov-19 factors value, Vul_Cov-19 factors value, and Unxch_Cov-19 factors are the dependent variable which is affected by independent variable disturbance impact of personal productivity (D_PP) variable. The coronavirus outbreak is first and foremost a human tragedy, affecting hundreds of thousands of people. It is also having a growing impact on the global economy. The COVID-19 outbreak affects all segments of the population and is particularly detrimental to members of social groups in the most vulnerable situations, continues to affect populations, including people living in poverty situations, older persons, persons with disabilities, youth, and indigenous peoples. Early evidence indicates that the health and economic impacts of the virus are being borne disproportionately by poor people. The virus is primarily spread between people during close contact often through small droplets produced by coughing, sneezing, or talking. While these droplets are produced when breathing out, they usually fall to the ground or onto surfaces rather than being infectious over long distances. People may also become infected by touching a contaminated surface and then touching their eyes, nose, or mouth. The virus can survive on surfaces for up to $72 \mathrm{~h}$. It is most contagious during the first 3 days after the onset of symptoms, although spread may be possible before symptoms appear and in later stages of the disease [17]. Thr_Cov-19 factors value has a significant impact on D_PP. The virus is primarily spread between people during close contact often through small droplets produced by coughing, sneezing, or talking. While these droplets are produced when breathing out, they usually fall to the ground or onto surfaces rather than being infectious over long distances. The vulnerability part occurred when people become infected by touching a contaminated surface and then touching their eyes, nose, or mouth. The virus can survive on surfaces for up to $72 \mathrm{~h}$. It is most contagious during the first 3 days after the onset of symptoms, although spread may be possible before symptoms appear and in later stages of the disease.

\section{ACKNOWLEDGMENTS}

We gratefully thankful to the University Malaysia Kelantan including all the faculties. We thank all the Chittagong Diabetic General Hospital, Chittagong, Bangladesh, and all the helping hands who support and help us in every step of our study.

\section{AUTHORS' CONTRIBUTIONS}

All the authors had equal contribution on this study. Task was separated to all authors according to their expertise on specific topic. Dr. Shirin Afroz generated the research topic for this study and reviewed relevant literatures. Dr. Shekh Abdullah-Al-Musa Ahmed and Dr. Nik Zulkarnaen 
Table 3: Discriminate validity

\begin{tabular}{|c|c|c|c|c|}
\hline & $\begin{array}{l}\text { Disturbance impact of } \\
\text { personal productivity } \\
\text { (D_PP) }\end{array}$ & $\begin{array}{l}\text { Threat of COVID-19 } \\
\text { (Thr_Cov-19) }\end{array}$ & $\begin{array}{l}\text { vulnerability } \\
\text { of COVID-19 } \\
\text { (Vul_Cov-19) }\end{array}$ & $\begin{array}{l}\text { Unexpected change in } \\
\text { society due to COVID-19 } \\
\text { attacks (Unxch_Cov-19) }\end{array}$ \\
\hline Disturbance impact of personal productivity (D_PP) & 0.745 & 0.623 & & \\
\hline Threat of COVID-19 (Thr_Cov-19) & 0.645 & 0.572 & 0.712 & \\
\hline vulnerability of COVID-19 (Vul_Cov-19) & 0.823 & 0.661 & 0.622 & \\
\hline $\begin{array}{l}\text { Unexpected change in society due to COVID-19 } \\
\text { attacks (Unxch_Cov-19) }\end{array}$ & 0.732 & 0.639 & 0.526 & \\
\hline
\end{tabular}

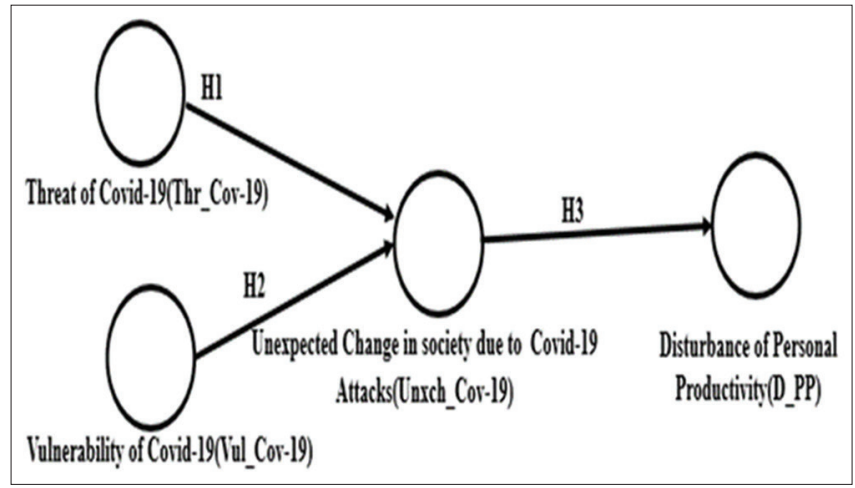

Fig. 2: The hypothesis relationship of the risk factors of disturbance of personal productivity due to COVID-19 attacks in the society

Bin Khidzir were the designer for the study. He designed methodology and analysis plan for the study and analyzed all the outcomes of the study and they also contributed on overall write up.

\section{COMPETING INTEREST}

Authors declare that they have no competing interest.

\section{FUNDING}

Authors declare that there was no any funding received for conducting this study.

\section{REFERENCES}

1. Adrian M. Running the risk IT-more perception and less probabilities in uncertain systems. Inf Comput Secur 2017;25:45-59.
2. Andrea R, Giuseppe R. COVID-19 and Italy: What next? J Lancet 2020;395:10231.

3. Ericson CA. Hazard Analysis Techniques for System Safety. United States: John Wiley \& Sons; 2016.

4. Ensheng D, Hongru D, Lauren G. An interactive web-based dashboard to track COVID-19 in real time. J Lancet Infect Dis 2020;38:347-9.

5. Hussin A, Siddappa N. The epidemiology and pathogenesis of coronavirus disease (COVID-19) outbreak. J Autoimmun 2020;109:24-35.

6. Nik Z, Shekh A. Towards fact-based digital forensic evidence collection methodology. Int J Inf Secur Res 2019;9:67-79.

7. Nik Z, Shekh A. Legal protection of intellectual property rights (IPR) in Bangladesh. Int J Law Gov Commun 2018;3:71-89.

8. Nik Z, Shekh A, Tan T. Viewpoint of probabilistic risk assessment in artificial enabled social engineering attacks. BITARA Int J Civiliz Stud Hum Sci 2018;1:32-9.

9. Puja M, Daniel F, Michael B, Emilie S. COVID-19: Consider cytokine storm syndromes and immunosuppression. J Lancet 2020;395:1133-6.

10. Roy A, Hans H, Don K. How will country-based mitigation measures influence the course of the COVID-19 epidemic? J Lancet 2020;395:931-4.

11. Shekh A, Nik Z. An exploratory factor analysis of AI enabled social engineering (SoE) attacking risk in higher learning institute. J Mass Commun J 2018;15:32-40.

12. Thirumalaisamy P, Christian G. The COVID-19 epidemic. J Trop Med Int Health 2020;25:278-80.

13. Wiebke A. Agents, Trojans and tags: The next generation of investigators. Int Rev Law Comput Technol 2009;23:99-108.

14. Yin RK. Case Study Research Design and Method Newbury Park. United States: SAGE Publications; 1984.

15. Ying L, Albert A, Wilder S, Joacim R. The reproductive number of COVID-19 is higher compared to SARS coronavirus. J Travel Med 2020;27:34-40.

16. Zheng YY, Ma YT, Zheng JY. COVID-19 and the cardiovascular system. Nat Rev Cardiol 2020;37:89-92.

17. Zhe X, Lei S, Yijin W, Jiyuan Z. Pathological findings of COVID-19 associated with acute respiratory distress syndrome. J Lancet Respir Med 2020;8:420-4. 